\title{
Corrigendum to "A Liquid-Based Cytology System, without the Use of Cytocentrifugation, for Detection of Podocytes in Urine Samples of Patients with Diabetic Nephropathy"
}

\author{
Moritsugu Kimura $\left(\mathbb{D},{ }^{1}\right.$ Masao Toyoda $\left(\mathbb{D},{ }^{1}\right.$ Nobumichi Saito, ${ }^{1}$ Noriko Kaneyama $\left(\mathbb{D},{ }^{1}\right.$ \\ Han Miyatake $\mathbb{D D}^{1}$ Eitaro Tanaka $\mathbb{D},{ }^{1}$ Hirotaka Komaba $\mathbb{D}^{1},{ }^{1}$ Masanori Hara, \\ and Masafumi Fukagawa $\mathbb{D}^{1}$ \\ ${ }^{1}$ Division of Nephrology, Endocrinology and Metabolism, Department of Internal Medicine, Tokai University School of Medicine, \\ Isehara, Japan \\ ${ }^{2}$ Iwamuro Health Promotion Center, Niigata, Japan \\ Correspondence should be addressed to Masao Toyoda; m-toyoda@is.icc.u-tokai.ac.jp
}

Received 18 June 2020; Accepted 22 June 2020; Published 30 June 2020

Copyright (c) 2020 Moritsugu Kimura et al. This is an open access article distributed under the Creative Commons Attribution License, which permits unrestricted use, distribution, and reproduction in any medium, provided the original work is properly cited.

In the article titled "A Liquid-Based Cytology System, without the Use of Cytocentrifugation, for Detection of Podocytes in Urine Samples of Patients with Diabetic Nephropathy" [1], the authors would like to clarify why the SurePath ${ }^{\mathrm{TM}}$ method was used in urine from diabetic nephropathy patients. The following text should be replaced with the addition of two missing references, 26 and $27[2,3]$ :

Original:

A similar technique of liquid-based cytology currently used in the cytodiagnosis of cervical cancer has replaced the conventional Pap smear cytology [25]. Our method using SurePath ${ }^{\mathrm{TM}}$ is simple and improved the detection of podocytes in urine samples.

Revised:

A similar technique of liquid-based cytology currently used in the cytodiagnosis of cervical cancer has replaced the conventional Pap smear cytology [25]. Additionally, a similar approach has been used to detect WT-1-positive cells in kidney disease [26], and its application in the diagnosis of kidney disease has also been studied [27]. Our method using SurePath $^{\mathrm{TM}}$ is simple and is expected to improve the detection of podocalyxin-positive podocytes in urine samples compared with our conventional method.

\section{References}

[1] M. Kimura, M. Toyoda, N. Saito et al., "A liquid-based cytology system, without the use of cytocentrifugation, for detection of podocytes in urine samples of patients with diabetic nephropathy," Journal of Diabetes Research, vol. 2019, Article ID 9475637, 7 pages, 2019.

[2] H. Ohsaki, T. Sofue, K. Kawakami et al., "WT1 immunoenzyme staining using SurePath ${ }^{\mathrm{TM}}$ processed urine cytology helps to detect kidney disease," Cytopathology, vol. 27, no. 1, pp. 4349, 2016.

[3] T. Fujita, T. Sofue, M. Moritoki et al., "Urinary WT1-positive cells as a non-invasive biomarker of crescent formation," Cytopathology, vol. 28, no. 6, pp. 524-530, 2017. 